\title{
A Performance Evaluation of Call Resolution Oriented Routing Rules to Enhance Resolution Rates
}

\author{
Mughele Ese Sophia \\ Department of Computer Science, \\ Delta State School of Marine \\ Technology Burutu, \\ Delta State \\ Nigeria
}

\author{
Stella C. Chiemeke \\ Professor of Computer Science and \\ Director of Intellectual Property and \\ Technology Transfer Office (IPTTO), \\ University of Benin, \\ Benin City, \\ Edo State Nigeria
}

\begin{abstract}
In contemporary society, satisfying customer's needs has become a phenomenon seen to be highly inevitable for business that wants to survive in this era of high competition amidst the global financial crisis. A customer's experience during a service encounter consist of two parts namely: the time spent waiting for the service and the service itself. The nature of the service, which is the resolution status, is a major key performance indicator (KPI) to measure the success of a call center. The challenge in the traditional call centres in Nigeria, is the ineffective resolution of calls which causes customers to call back immediately after an interaction with a call center agent. This is because the issue was not resolved in the previous encounter. The purpose of this research is to evaluate the performance results of three Call Resolution (CR) routing rules, using data collected from the call center of a telecommunication organisation Nigeria. The evaluation was conducted using simulation techniques. A sample of 2,000 calls was used for the simulation. Java programs were developed for each of the routing rules because they vary from one approach to another in operation. Results from the simulation gave the performance of all routing rules for CR, non $\mathrm{CR}$, percentages of resolved call and call backs. From the result, we observed that the higher the resolved calls the lower the rate of call backs and vice versa. We also observed that out of the proportion of unresolved calls, a particular number of customers did not call back. The result from the study gave the optimal routing rule to be the Shortest Queue Routing (SQR), which proffered an enhanced call resolution rate and a very low call back rate. The implementation of the SQR as the optimal routing rule, will improve performance of call center management with respect to enhanced $\mathrm{CR}$ and reduced call backs.
\end{abstract}

\section{Keywords}

Call Center, Call Resolution, Call Backs, Routing Rules, Simulation

\section{INTRODUCTION}

A customer is faced with two challenges when calling a call center, firstly, the time spent on the queue waiting for service and secondly, the nature of the service itself. Call centres give priority to the two criteria with emphasis on one more than the other. Those that place more emphasis on time spent waiting for the service are more concerned with reducing the average time involved in handling a call while those that are concerned with the service itself aims at effective resolution of customer issues. Call centre can be defined as any group whose business is talking to customers or prospective customers through the telephone. According to Brizola et al (2001), a call centre is a system that offers complete management of all communication channels between a business and its customers, optimizing polices, eliminating duplicated work and making better use of time. The call centre service has grown a great deal with its application in all sectors of the economy. It serves as a primary contact between businesses and clients.

Most research in the domain of call canter focuses on waiting time which is a result of queue. The issue of queuing has been a subject of scientific debate, for there is no known society that is not confronted with the problem of queuing. Wherever there is competition for limited resources queuing is likely to occur. As a result, capacity planning and call routing software systems strive to minimize costs while achieving self-imposed service level constraints, such as "average wait in queue less than 15 seconds". These traditional approaches do not consider, however, the quality of answers provided by the call center agent. Beyond the challenge of queue in a call center is the problem of call resolution. This is the ability of a call center agent to resolve the customer's issue effectively the very first time the customer calls the call center.

Low quality of service has a significant impact on the call center operations besides customer defection: As dissatisfied customers call back for more help for the same problem, the load on the system increases. First Call Resolution (FCR) is perhaps the most powerful call center metric. FCR measures the percentage of customer issues resolved the first time. A call held waiting in the queue that ended with solving the customer issue is better than a call that got instantly connected to an agent who could not properly help the customer. A call center maintaining a good FCR rate receives a small amount of calls coming from customers who have to call back because their issue was not resolved the first time. The call center avoids a significant cost due to higher call volume, increased operating expenses, and dissatisfied customers.

For a call centre that is primarily focused on call resolution, it seems optimal to route each call type to the agent who can handle it the best, therefore holding such calls in queue even if other agents are not busy, until the agent who can handle such calls properly become available later. However, in an environment where there is significant variability across different agents' resolution probabilities, routing rules that are based solely on these rates are likely to lead to long queues. Determining agent's resolution ability across the various agent groups and determining the routing rule that is appropriate to route calls to agent according to the order of their resolution ability will definitely, reduce undue burden on some agent groups while other agent groups experience low levels of utilization and excessive idle time. Our research is motivated by the fact that a customer will have to call a call 
centre more than three times on one issue or problem, because the issue was not effectively resolved in each of the session the call was made, and at times the issue may not be resolved.

The call resolution probability is directly related to a customer's perception of call quality which depends on the call center agent's understanding of the customer's needs, courtesy, and competency (Zeithaml et al.1993). V'ericourt and Zhou (2004) suggested that a call center agent's call resolution probability is often highly correlated with his/her call speed (defined as the service rate). On the one hand, the correlation could be negative. Due to very high turnover rates and long training lead-time in this industry (Gans and Zhou, 2002), some call centers are pressed to make the most use of their agents. In this study, a performance evaluation on existing rules for call resolution routing rules was carried out. This evaluation was conducted by simulating each of the routing rules and using raw data collected from a foremost telecommunication organization in Nigeria. A collection of Java program was deployed for the simulation.

\section{LITERATURE REVIEW}

Armony (2005) stated that for a call centre to reduce waiting lines with emphasis on the reduction of time spent, its best to route calls to agents who can handle customer issues the fastest, sometimes even holding a call in queue to wait for that agent than routing the call to a slower agent. This might lead to further increase in congestion, repeat calls from unreceptive issues and undue burden on some agents. Vericourt et al. (2005), states that for a call centre to reduce waiting lines, emphasis should be on the service itself that is; call resolution. Its best to route calls to agents who resolve customer issues, sometimes holding a call in queue to wait for such agent. This might also lead to increase in congestion and undue burden on some agents. Once a customer received service from a contact center agent regarding a particular problem, a subsequent call from such a customer regarding that same problem is a clear indication that the problem was not properly resolved in the previous service encounter. The inability of the agent to resolve this problem will obviously lead to customer's dissatisfaction. Thus, First Call Resolution rates are very important customer-centric operational metrics in most organization. Although 'these have been largely absent from the academic literature on call centre operations'. As data collection and analysis technologies for accurately measuring Resolution Probability values begin to emerge, call centre managers are increasingly focused on managing the Call Resolution and First Call Resolution metrics. Higher Call Resolution and First Call Resolution rates result in reduced system congestion (due to decreased call-backs and hence lower total call rates) and subsequently lower staffing costs. As such, these metrics have been attracting more attention from call centre leaders.

First call resolution is the proper addressing of the customer's needs the first time they call, thereby eliminating the need for the customer to follow up with the second call. Resolving customer issues the first time is the most appropriate way to improve customer satisfaction. Studies reveal that caller satisfaction ratings will be $5-10 \%$ lower when a second call is made for the same issue. Eliminating the need for customers to wait on hold not only increases customer satisfaction, it also reduces costs on call centers. This really is a best solution that many call centers are missing out on. When callers are put on hold, this increases telecommunication costs and most likely, many caller will abandon the queue or rather end the conversations. In fact, $60 \%$ of customers will abandon a call after one minute of waiting on hold. Instead, call centers should offer customers the option to receive a call-back rather than been kept on hold on the queue. In Mehrotra et al. (2012), the rules that explicitly emphasize CR rates are:

1. Shortest Queue Routing (SQR): A call of a particular type that arrives when multiple agents are free will be routed to an agent from the group that has the shortest queue for that call type.

2. Probabilistic Routing (PR): A call of a particular type that arrives when multiple agents are free will be routed to an agent from the group that has the highest resolution probability for that call type.

3. Relative Resolution Probability Routing (RRPR): a call of a particular type that arrives when multiple agents are free will be routed to an agent from the group that has the highest relative resolution probability for that call type.

Call routing is the sequence of path taken to convey a customer's call to a service agent. Call routing also known as call distribution relates to a set of rules which are applied to isolate the most appropriate resource for a specific call. Call routing is experience by the customer as being guided through a decision tree. By progressing through that tree the system provides information to and collects user inputs from the caller. The corresponding realization is often referred to as routing path. However having reached the leaf of the decision tree, the collected information is considered as being sufficiently complete and call distribution takes over to determine the most appropriate agent based on agent properties, user input and system load to route the call.

All routing techniques or algorithms used in call distribution follows a baseline routing rule which serves as a benchmark for routing cells (Mehrotra, 2009). The benchmark routing rule usually followed is the first-come, first serve or longest wait rule. Here the rule states that the first customer to arrive on a queue or the customer that has waited the longest on the queue and it follows the sequence until all calls are attended to.

All call centres have an organizational structure and understanding the structure can be of great importance in determining the most effective structure that suite the current and planned operation (Klenkei 2006). The most common approaches in handling call flow in a call centre is either to use the cross-grained agent approach or the specialized trained agent approach.

a. Cross-Trained Agent Approach:- The approach is a situation whereby an agent is trained in his colleague's duties. In a situation where there are different types of call, agents are trained to handle the different types of call. This approach represents flexible capacity where every agent is capable of attending to the different types of call available.

b. Specialized - Trained Agent Approach:- The approach is a situation whereby an agent is trained to attend only to a particular type of call. This enables the agent to be faster in attending to such calls and there is a particular agent for each particular type of call.

Given the size of the call center industry and the complexity associated with its operations, call centers have emerged as a fertile ground for academic research. Hart et al. (2006) provides a complete review of articles on FCR while also pointing out the importance of measuring and using FCR. 
Resolving customer queries the first time around is a commonly shared goal. A company's business context, human resources strategy, supporting technology and budget constraints influences this Key Performance Indicator (KPI) in many ways, and makes First Call Resolution (FCR) a difficult measure to benchmark. The study established the differing views on the value and measurement of FCR, identifies the main factors affecting FCR and the relationships among these factors, and relates results in a South African context to academia and practitioner.

Véricourt and Zhou (2005) also discovered that traditional research on routing in queuing systems usually ignores service quality related factors. Customers call back when their problems are not completely resolved by the customer service representatives. They used a Markov decision process formulation to obtain analytical results and insights about the optimal routing policy that minimizes the average total time of call resolution, including callbacks. They establish the fact that: for each call, both the call resolution probability $(\mathrm{P})$ and average service time $(1 / \mathrm{m})$ are customer service dependent.

Stanley et al (2008) posited that in a service base call center, the two key challenges are (i) Where should a call be routed to and (ii. Who should handle the call?) The researchers deployed base case FIFO approach for the simulation modeling to analyze performance-based routing strategies in call centers. Their work shows the potential for significant improvements in call center performance especially, Average Speed to Answer (ASA), by using rules based on historic performance data such as Average call Handling Time (AHT) and First call Resolution (FCR) rates.

Dabrowski (2013) observed that the key performance indicators to measure call center performance are not effectively maximized. Metrics such as average speed of answer, cost per call, agent utilization rate, first contract resolution rate, customer satisfaction and aggregate call center performance. The researcher used CallLogic system to improve the fundamental call routing logic of the Northeast Utilities call centers. Although the findings of the CallLogic system lead to discoveries and ideas on how to improve the fundamental call routing logic of the Northeast Utilities call centers, the CallLogic project achieved high success in the average call handling time. The study only made mention of call Resolution rate and its impact on operational success.

The quality of service accessibility and customer waiting time are dominant performance measures (Vericourt and Zhou 2005). Hence capacity planning and call routing software system strive to minimize cost while achieving self imposed service level constraints, hence considering low average time waiting in queue, these approach do not consider the quality of service rendered to customers (Vericourt and Zhou 2005). Low quality of service has significant impact on the call center operations; this operational impact of service failure is often ignored by call center capacity planning and call routing management system. Their work was motivated by the fact that a major European telecommunications service provider discovered that customers needed to talk to more than three different agents before their problems are resolved.

Mehrotra et al (2012), maximizes CR routing rules as one the metrices for call center operational performance. They modelled an optimization problem that focus on call resolution, taking into cognisance the work of L'Ecuyer (2006) and Gans et al (2010) that minimized wait time routing rule. This study is focused on conducting a performance analysis on CR oriented routing rules using simulation process to determine optimality among the rules. This will improve the performance of call routing and enhance $\mathrm{CR}$, which further reduces call backs to the system network as a result of ineffective resolution of customer's issues.

\section{RESEARCH METHOD}

The research was conducted using data collected from a foremost telecommunication organization in Nigeria as a case for the study. A structured Interview was conducted at the organisation's headquarters Lagos in Nigeria. This was necessary for investigating their mode of operation and possible routing rules been adopted by their call centre. Three (3) personnel were interviewed at the call centre. The outcome of the interviews were relevant for the study, having understood the call centre operations, a further request was made for call centre data from its automated data logging system comprising of agent identity, calls attended to, call handling time, call status, etc. These data were used to test each of the three routing rules to determine their performance. A JAVA simulation program was designed for each of the routing rules using the data collected from the organisation. The result from the simulation gave the optimal rules for Call Resolution oriented routing rules.

\subsection{Model Approach}

In this model, we consider multiple call types (indexed by $\mathrm{i}=$ $1,2 \ldots \mathrm{y}$ ) and multiple agent groups (indexed by $\mathrm{j}=1,2 \ldots \mathrm{z}$ ). Calls of type $i$ arrive at a rate of $\wedge_{i}$. There are nj agents in group $\mathrm{j}$, with njعZ+ and each agent in group $\mathrm{j}$ serves call type $\mathrm{i}$ with rate $\mu \mathrm{ij}$. Here we allow agents to handle only a subset of all the call types. If agent group $\mathrm{j}$ is not capable of handling call type $\mathrm{i}$ then $\mu \mathrm{ij}=0$. When $\mu \mathrm{ij}>0$ we say there is a "match" between call type $i$ and agent group $j$. In addition, we assume independence of past history each agent of group $\mathrm{j}$ has a resolution probability for each call of type i of pije $[0,1]$.

In the routing rules, $\mathrm{Qi}(\mathrm{t})$ represents the number of type $\mathrm{i}$ customers waiting for service at time $t$ and $f j(t)$ be the number of available agents of type $\mathrm{j}$ who are free at time $t$, where $0 \leq \mathrm{fj}$ (t) $\leq \mathrm{nj}$, for all $\mathrm{j}$, t.

Formally, we use the term "routing rule" to mean both the logic that determines to which agent group an arriving call is assigned if there are no calls in queue and agents from multiple groups are free as well as the logic that determines which call an agent is assigned to handle when he/she becomes free when calls from more than one type are in queue waiting for service.

\subsection{Models for Existing Routing Rules}

As adapted from Mehrotra et al. (2012), "the benchmark routing rule is the First-Come-First-Served/Longest-Wait $(\mathrm{FCFS} / \mathrm{LW})$ rule", because this is the routing rule deployed in most telecommunications call centers and in majority of other call centers. Our work focuses on models for CR oriented rules which we specify as follows.

\subsubsection{Resolution Probabilistic Routing Rules}

Call resolution rate is a major key performance Indicator (KPI) in call center operation, some call centres may place a much higher priority on CR rates. Thus, in this study we describe routing rules that explicitly emphasize CR rates. (Garcia et al 2012, Aksin et al 2007, and Vericourt and Zhou 2005)

\section{Shortest Queue Routing (SQR):}

A call of a particular type that arrives when multiple agents are free will be routed to an agent from the group that has the shortest queue for that call type. 
Let $\mathrm{Qi}(\mathrm{t})$ represents the number of type i customers waiting for service at time $t$ and

Let $f j(t)$ be the number of available agents of type $j$ who are free at time $t$,

Where $0 \leq \mathrm{fj}(\mathrm{t}) \leq \mathrm{nj}$, for all $\mathrm{j}$, t.

Let Multiple call types be indexed by $\mathrm{i}=1,2$...I and

Let Multiple agent groups be indexed by $\mathrm{j}=1,2$...J.

Calls of type $i$ arrive at a rate of $\wedge_{i}$

There are nj agents in group $\mathrm{j}$, with nj\&Z+

Each agent in group $\mathrm{j}$ serves call type $\mathrm{i}$ with rate $\mu \mathrm{ij}$

/Here we allow agents to be trained to handle only a subset of all the call types/

If agent group $\mathrm{j}$ is not capable of handling call type I then $\mu \mathrm{ij}$ $=0$

When $\mu \mathrm{ij}>0$ we say there is a "match" between call type $\mathrm{i}$ and agent group

/In addition, we assume independent of past history/

Each agent of group $\mathrm{j}$ has a resolution probability for each call of type i of pije $[0,1]$.

When an agent of group $\mathrm{j}$ becomes free,

Select argmaxi:Qi(t) $>0\{$ pij $\mu \mathrm{ij}-\operatorname{maxk} \neq$ jpik $\mu \mathrm{ik} \mid \mu \mathrm{ij}>0\}$

/that is, an agent coming free will choose the matching call type for which she has the highest relative effective service rate/

Similarly, if an arriving call of type i finds no calls of that type waiting for service and agents of one or more matching group available, select a matching agent group $\mathrm{j}$

Where $\mathrm{j}=\operatorname{argmaxj}: \mathrm{fj}(\mathrm{t})>0\{$ pij $\mu \mathrm{ij}-\operatorname{maxk} \neq \mathrm{jpik} \mu \mathrm{ik} \mid \mu \mathrm{ij}>0\}$

/that is, a call of a particular type that arrives when multiple matching agents are free will be routed to an agent from the matching group that has the highest relative effective service rate for that call type also referred to as the shortest queue for that call type/

\section{Probabilistic Routing (PR):}

A call of a particular type that arrives when multiple agents are free will be routed to an agent from the group that has the highest resolution probability for that call type.

Let $Q_{i}(t)$ represents the number of type $i$ customers waiting for service at time t and

Let $f_{j}(t)$ be the number of available agents of type $j$ who are free at time $t$

Where $0 \leq f_{j}(t) \leq n_{j}$, for all $j, t$.

Let Multiple call types be indexed by $i=1,2 \ldots I$ and

Let Multiple agent groups be indexed by $j=1,2 \ldots J$.

Calls of type $i$ arrive at a rate of $\wedge_{i}$.

There are $n_{j}$ agents in group $j$, with $n_{j} \varepsilon Z^{+}$

Each agent in group $j$ serves call type $i$ with rate $\mu_{i j}$

/Here we allow agents to be trained to handle only a subset of all the call types/
If agent group $j$ is not capable of handling call type I then $\mu_{i j}$ $=0$

When $\mu_{i j}>0$ we say there is a "match" between call type $i$ and agent group

/In addition, we assume independent of past history/

Each agent of group $j$ has a resolution probability for each call of type $i$ of $p_{i j} \varepsilon[0,1]$.

When agent $j$ becomes free, select $\operatorname{argmax}_{i: Q i(t)>0\{}\left\{p_{i j} \mid \mu_{i j}>0\right\}$

/that is, that agent will be assigned a call of the type that she is most likely to resolve, regardless of waiting times and queue lengths/

Similarly, if an arriving call of type i finds no calls of that type waiting for service and agents of one or more group available, assign that call an agent of group $\mathrm{j}$,

Where $j=\operatorname{argmax}_{j: f(t)>0\{}\left\{p_{i j} \mid \mu_{i j}>0\right\}$

/that is, a call of a particular type that arrives when multiple agents are free will be routed to an agent from the group that has the highest resolution probability for that call type/ (Mehrotra et al, 2012)

\section{Relative Resolution Probability Routing (RRPR):}

a call of a particular type that arrives when multiple agents are free will be routed to an agent from the group that has the highest relative resolution probability for that call type.

Let $Q_{i}(t)$ represents the number of type $i$ customers waiting for service at time $t$ and

Let $f_{j}(t)$ be the number of available agents of type $j$ who are free at time $t$,

Where $0 \leq f_{j}(t) \leq n_{j}$, for all $j, t$.

Let Multiple call types be indexed by $i=1,2 \ldots I$ and

Let Multiple agent groups be indexed by $j=1,2 \ldots J$.

Calls of type $i$ arrive at a rate of $\wedge_{i}$.

There are $n_{j}$ agents in group $j$, with $n_{j} \varepsilon Z^{+}$

Each agent in group $j$ serves call type $i$ with rate $\mu_{i j}$

/Here we allow agents to be trained to handle only a subset of all the call types/

If agent group $j$ is not capable of handling call type I then $\mu_{i j}$ $=0$

When $\mu_{i j}>0$ we say there is a "match" between call type $i$ and agent group

/In addition, we assume independent of past history/*

Each agent of group $j$ has a resolution probability for each call of type $i$ of $p_{i j} \varepsilon[0,1]$.

When agent $j$ becomes free, select $\operatorname{argmax}_{i: Q i(t)>0}\left\{p_{i j}-\right.$ $\left.\max _{k \neq j} p_{i k} \mid \mu_{i j}>0\right\}^{*}$

/that is, that agent will be assigned a call of the type that she is relatively most likely to resolve/

Similarly, if an arriving call of type i finds no calls of that type waiting for service and agents of one or more group available, assign that call an agent of group $\mathrm{j}$,

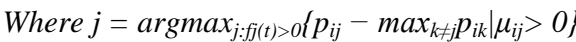


/that is, a call of a particular type that arrives when multiple agents are free will be routed to an agent from the group that has the highest relative resolution probability for that call type/ (Mehrotra et al, 2012)".

\subsection{Simulation Procedure}

Having presented a diverse set of routing rules, we therefore, determine how well each of these routing rules performs. In particular, we define the performance of these routing rules in terms of the key performance metrics of aggregate call resolution $(\mathrm{CR})$ rate. For the call center simulation process, we conducted an extensive simulation study based on data obtained from the organinisation earlier mentioned call centre. Below we describe the operational input data, the simulation modelling platform, the program structure and then present and discuss the results from the simulation. The simulation contained as inputs the date and time of the call, the unique ID number for the agent who handled the call, the Call Type for that call, and the resolution status of the call. We used only a subset of the call types and agents to ensure that the run times for our simulations were fast enough to conduct extensive numerical experiments. The process of selecting and preparing the data to support our numerical experiments during implementation included the following:

1. Selection of Call Types: The number of call types is a significant driver of simulation times; hence we considered the largest call types.

2. Selection of Agents: We restricted the number of agents in our model to include only those agents who can handle a certain amount of calls.

3. Agent clustering: There are a total of 175 agents, and they are clustered into 8 groups.

The numbers of agents in each group are given in Table 1.

Table 1: Total number of Agents in Agent groups

\begin{tabular}{|l|l|l|l|l|l|l|l|l|}
\hline $\begin{array}{l}\text { Agent } \\
\text { Group }\end{array}$ & 1 & 2 & 3 & 4 & 5 & 6 & 7 & 8 \\
\hline $\begin{array}{l}\text { Number } \\
\text { in Group }\end{array}$ & 35 & 35 & 15 & 10 & 10 & 25 & 30 & 15 \\
\hline
\end{tabular}

(Source: Field study, 2015)

4. Arrival Rate Selection: For our numerical experiments, we chose arrival rates for each of the call types to maintain the same relative proportion of expected calls of call found in the database. The total new arrival (not including call backs of unresolved calls) is set at 2000calls/hour. This defines the proportion of the calls that goes into queues.

Each routing rule was used independently with the collected data to simulate the call centre operation. Simulation was carried out by using the data gathered from the above call centre to estimate parameters needed to characterize the model. At the end of each simulation analysis, it is important to note that the performance of this system is defined in terms of the CR rate, and that this output metrics depend not only on the actual numerical values of the input parameters but also on choice of the routing rule that is used to determine which call types are handled by which agents under what conditions. The overall CR for each routing rule is the weighted average over all agent groups and call types. For example, Table 3 shows a sample result of our simulation analysis.

\section{IMPLEMENTATION AND RESULT}

The input data in Table 2, shows the various service type, number of calls offered, analysis of the number of calls answered, abandoned, average speed of answer, average talk duration and other report from the calls offered. The Java program developed is a standalone application. On executing the program, the screenshots showing the simulation processes are shown in Figures $1-3$ in the Appendix.

Table 2: Input call type data for simulation

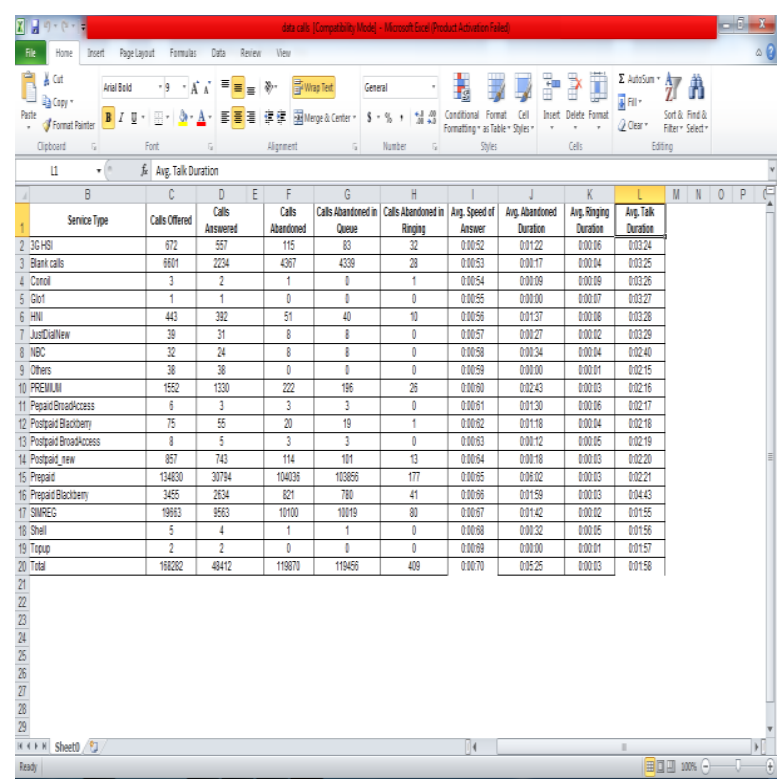

\subsection{Simulation Results}

Table 3 shows the call resolution and non call resolution and percentages of resolved and call backs for each of the routing rules. From the simulation result, SQR has the highest CR rate than PR and RRPR and RRPR has the least performance. The simulation result also shows the percentages of resolved calls and call backs. The resolution probability of each of the routing rules is a reflection of the values on the columns for percentages.

Table 3: Weighted Average Results for evaluation obtained from simulation Analysis

\begin{tabular}{|c|c|c|c|c|c|c|}
\hline $\begin{array}{c}\text { RUL } \\
\text { ES }\end{array}$ & CR & $\begin{array}{l}\text { Non } \\
\text { CR }\end{array}$ & $\begin{array}{c}\text { RESOLVE } \\
\text { D CALLS }\end{array}$ & $\begin{array}{c}\text { CALL } \\
\text { BACKS }\end{array}$ & $\begin{array}{c}\% \\
\text { resolv } \\
\text { ed } \\
\text { calls }\end{array}$ & $\begin{array}{l}\text { \% Call } \\
\text { backs }\end{array}$ \\
\hline SQR & 1795 & 205 & $\begin{array}{l}0.49861111 \\
1\end{array}$ & $\begin{array}{l}0.056944 \\
444\end{array}$ & $\begin{array}{l}83.101 \\
85185\end{array}$ & $\begin{array}{l}9.49074 \\
0741\end{array}$ \\
\hline PR & 1775 & 225 & $\begin{array}{l}0.49305555 \\
6\end{array}$ & 0.0625 & $\begin{array}{l}82.175 \\
92593\end{array}$ & $\begin{array}{l}10.4166 \\
6667\end{array}$ \\
\hline $\begin{array}{l}\text { RRP } \\
\mathrm{R}\end{array}$ & 1685 & 315 & $\begin{array}{l}0.42361111 \\
1\end{array}$ & $\begin{array}{l}0.071944 \\
444\end{array}$ & $\begin{array}{l}77.948 \\
0110\end{array}$ & $\begin{array}{l}14.5519 \\
850\end{array}$ \\
\hline
\end{tabular}

Figure 4 depicts the graph deduced from simulation result for evaluation of the three routing rules, which determines the optimal as SQR. 


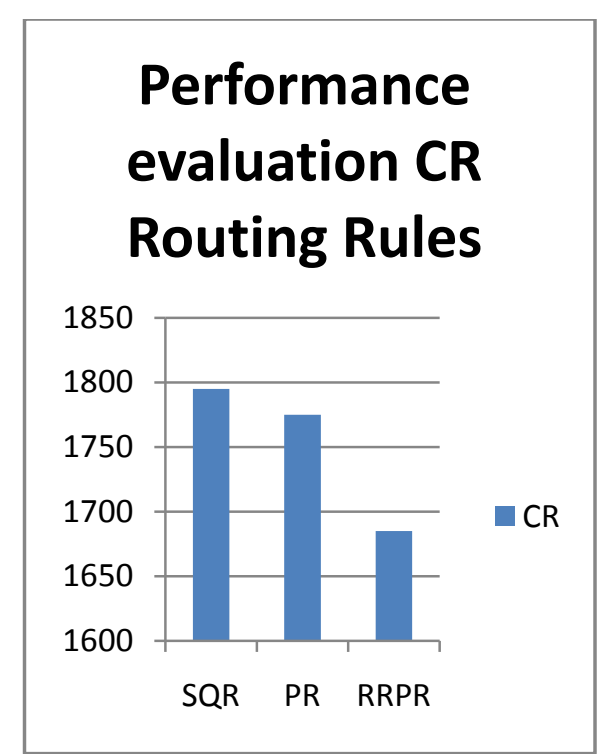

Figure 4: Performance evaluation obtained from simulation analysis

Figure 5 shows result for $\mathrm{CR}$ and non CR for routing rules, from the graph we observed that the higher the CR rate the lower the non CR rate and vice se versa.

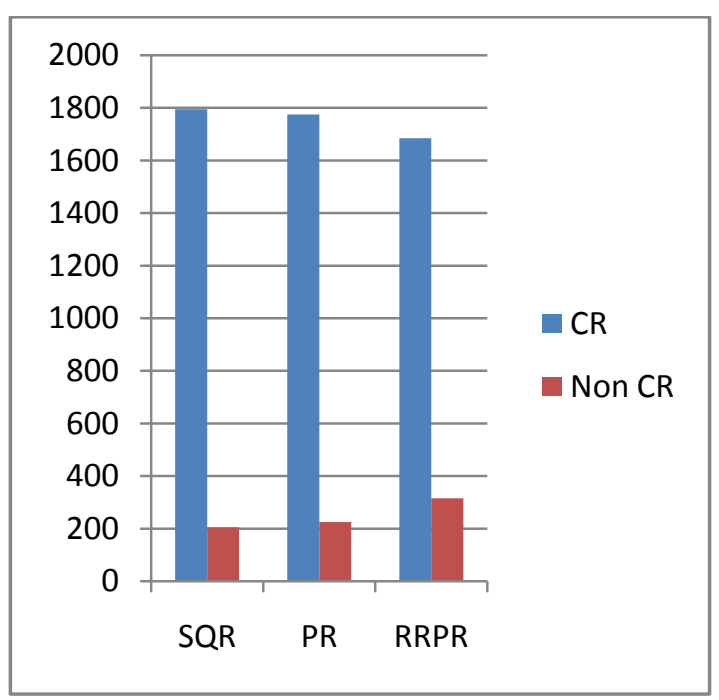

Figure 5: Evaluation Result from Simulation for CR and Non CR

Figure 6 shows percentages of resolve call for SQR $(83.10 \%)$ and call back $(9.49 \%)$. We observe that out of the 205 calls that were not resolved, $(7.41 \%)$ did not call. For PR percentage of resolved calls $(82.17 \%)$ and call back $(10.41 \%)$. Out of the 225 calls that was not resolved, $(7.5 \%)$ did not call back. The percentage of resolved calls for RRPR $(77.94 \%)$ and call backs $(14.55 \%)$. Out of the 315 unresolved calls (7.51\%) did not call back.

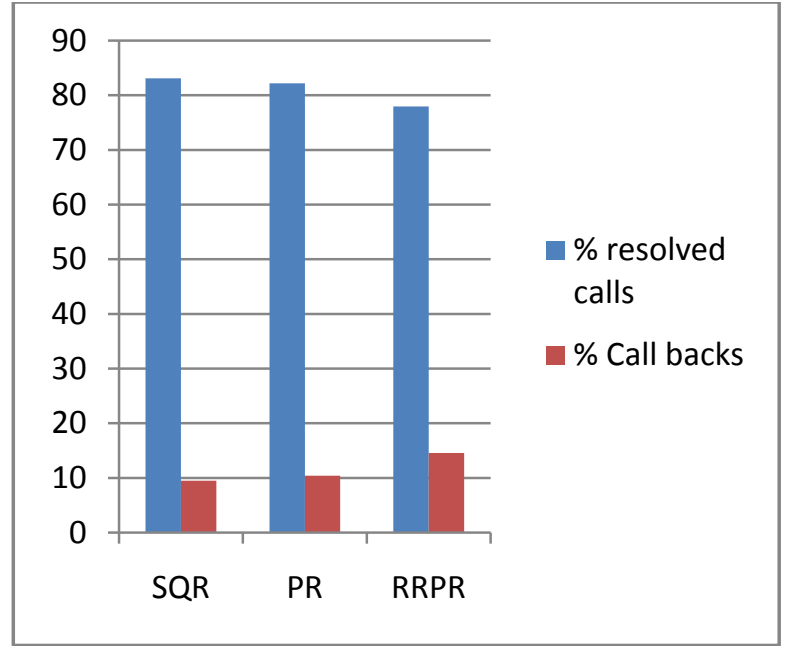

Figure 6: Result from Simulation for percentages of resolved calls and call backs

\section{CONCLUSION}

Most of the researches conducted in the domain of call centre operations/management are focused on reducing queues. From literature different authors have deployed various techniques to reduce waiting time on the queue from diverse perspective. Apart from reducing the Average Speed of Answer (ASA) for calls, there is also need for effective and enhanced call resolution which is a function of the ability of the call center agent, which is determined by the routing rule deployed by the call center operators. This study explored the various $\mathrm{CR}$ routing rules and conducted a performance evaluation on each of the rules. The evaluation was carried out using a collection of Java simulation library. Three CR rules were simulated using raw data collected from a telecommunication call center. Results from the simulation showed CR, non CR, percentage of resolved calls and call backs. The result also showed the performances of each of the routing rules on the variables measured. Result from the study established that SQR performs better than other rules evaluated. Further study can be conducted to consider environments with multiple call types, when there are also clear issues about which agents to train to handle which types of calls when both customer waiting times and call resolution rates are considered. While there has been a significant amount of research on skill-based routing and agent pooling, research can be conducted to consider the impact of such rules on $\mathrm{CR}$ rates when different agent groups have different Average Handling Time (AHT) and Resolution Probability (RP) values for different call types. Similarly, we have also taken the number of agents of each group as a timeindependent input into our model, though in practice these staffing levels are a function of an underlying scheduling model. Thus, another important related research area is incorporating RP (as inputs) and CR rates (as outputs) into call forecasting and agent scheduling models.

\section{REFERENCES}

[1] Aksin, Z. Armony, M. and Mehrotra. V. (2007) The modern call-centre: A multi-disciplinary perspective on operations management research. Production and Operations Management, 16(6):665-688, NovemberDecember Available at http://www.stern.nyu.edu/om/ faculty/armony/research/CallCentreSurvey.pdf. 
[2] Armony .M (2005), Dynamic routing in large-scale service systems with heterogeneous servers. Queueing Systems, 51(3-4):287-329, December 2005.

[3] Brizola, N, Costa .S, Pazeto .T, and Freitas P. (2001). Planejamento de Capacidade de Call Center. In : ICIE, Flo-rianópolis

[4] Dabrowski .M. (2013), Business Intelligence In Call Centers. International Journal of Issue Computer and Information Technology (ISSN: 2279 - 0764) Volume 02-02, March 2013. www.ijcit.com

[5] Gans .N, and Zhou .Y. (2002), Managing Learning and Turnover in Employee Staffing.Operations Research, 50(6), Nov-Dec

[6] Gans, N., N. Liu, A. Mandelbaum, H. Shen, H. Ye. (2010). Service times in call centers: Agent heterogeneity and learning with Powering Applications-A Festschrift for Lawrence D. Brown, IMS Collections, Vol. 6. Institute of Mathematical Statistics, Beachwood, $\mathrm{OH}$, 99-123. some operational consequences. Borrowing Strength: Theory Powering Applications-A Festschrift for Lawrence D. Brown, IMS Collections, Vol. 6. Institute of Mathematical Statistics, Beachwood, $\mathrm{OH}$, 99-123.

[7] Garcia. D, Archer .T, Moradi .S, and Ghiabi .B (2012), Waiting in Vain: Managing Time and Customer Satisfaction at Call Centers. Science Research, http://dx.doi.org/10.4236/psych.2012.32030. Psychology 2012. Vol.3, No.2, 213-216 Published Online February 2012 in SciRes http://www.SciRP.org/journal/psych)

[8] Klenkei .M. (2006) Call center staffing : the complete, practical guide to workforce management. Nashville, Tenn.: Call Center School, 3. Print, 197 S. graph. Darst. $23 \mathrm{~cm}$, ISBN: 0974417904, 978-0-9744179-0-5

[9] L'Ecuyer. P. (2006), Modelling and optimization problems in contact centres. Proceedings of the Third International Conference on the Quantitative Evaluation of Systems - (QEST'06), pages 145-154, 2006.

[10] Mehrotra .V, Ross .K, Ryder .G and Zhou .Y (2012), Routing to Manage Resolution and Waiting Time in Call Centers with Heterogeneous Servers. MANUFACTURING \& SERVICE OPERATIONS MANAGEMENT Vol. 14, No. 1, Winter 2012, pp. 6681 ISSN 1523-4614 (print). ISSN 1526-5498 (online) http://dx.doi.org/10.1287/msom.1110.0349 @2012 INFORMS

[11] Zeithaml .Z, Parasuraman .A and Berry .L (1993) The nature and determinants of customer expectations of service. Acad. Marketing Sci., 21(1),

\section{APPENDIX}

\section{Screenshots of Simulation Process}

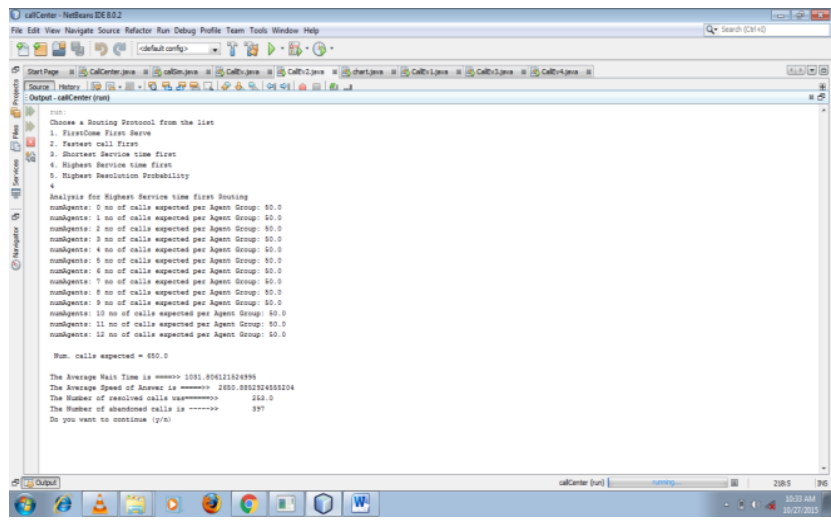

Figure 4.1: Screen shot of Simulation using Shortest Queue Routing (SQR)

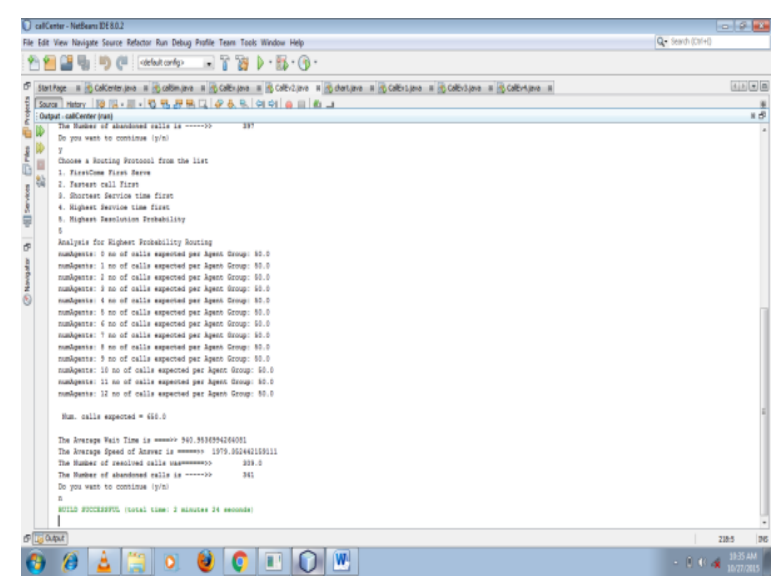

Figure 4.2: Screen shot of simulation analysis using Highest Resolution probability routing

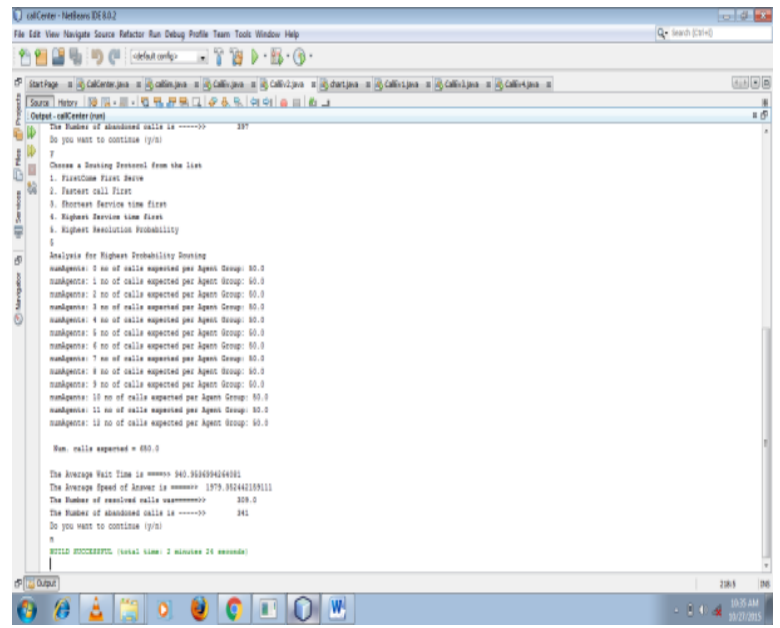

